\title{
A Comparison of Quantitative T2 Mapping on Cardiovascular Magnetic Resonance Imaging with Metaiodobenzylguanidine Scintigraphy and Left Ventricular Functional Recovery in Dilated Cardiomyopathy: A Retrospective Pilot Study
}

\author{
Tatsuro Ito ${ }^{1}$, Atsushi K. Kono ${ }^{2}$, Sachiko Takamine ${ }^{1}$, Mayumi Shigeru ${ }^{1}$, Shumpei Mori ${ }^{1}$, \\ Tomofumi Takaya ${ }^{1}$, Sei Fujiwara ${ }^{1}$, Tatsuya Nishii ${ }^{2}$, Hideyuki Shiotani ${ }^{3}$, \\ Kazuro Sugimura $^{2}$ and Ken-ichi Hirata ${ }^{1}$
}

\begin{abstract}
Objective Metaiodobenzylguanidine (MIBG) scintigraphy is used to assess heart failure (HF) severity and to predict cardiac functional recovery. Cardiovascular magnetic resonance (CMR) imaging has recently been used to diagnosis HF. We evaluated CMR T2 mapping and MIBG scintigraphy in dilated cardiomyopathy (DCM) patients.

Methods Consecutively, 22 DCM patients [aged 56.8 \pm 13.4 years; 6 women and 16 men; left ventricular ejection fraction (LVEF), 31.9 $\pm 10.7 \%$ ] who underwent T2 mapping and MIBG scintigraphy were retrospectively evaluated. Echocardiography results were recorded at baseline and the 6-month follow-up. Patients with an increased LVEF $\geq 15 \%$ between the 2 measures were considered to be responders. We measured each patient's T2 values and MIBG indices [the heart-to-mediastinum ratio $(\mathrm{H} / \mathrm{M})$ in the early phase, $\mathrm{H} / \mathrm{M}$ in the delayed phase, and the washout rate (WOR)] at baseline. We compared these values between the 12 responders and 10 non-responders.

Results The mean T2 value for all patients was $64.5 \pm 6.6 \mathrm{~ms}$. The mean values of early H/M, delayed H/M, and WOR were $2.06 \pm 0.25,1.94 \pm 0.35$, and $43.5 \pm 11.8 \%$, respectively. The T2 values were found to correlate with MIBG indices $(\mathrm{p}<0.05$ for all) and were lower in the responders than non-responders $(61.4 \mathrm{vs} .68 .1 \mathrm{~ms}$, $\mathrm{p}=0.013)$. MIBG indices were not significantly different.

Conclusion Our study shows that the T2 values correlated with the MIBG indices and were increased in non-responders. T2 mapping may be useful in assessing the cardiac function and functional recovery in DCM patients.
\end{abstract}

Key words: dilated cardiomyopathy, functional recovery, magnetic resonance imaging, metaiodobenzylguanidine, T2 mapping

(Intern Med 54: 2121-2128, 2015)

(DOI: 10.2169/internalmedicine.54.4493)

\section{Introduction}

Idiopathic dilated cardiomyopathy (DCM) is characterized by dilated ventricles and diminished cardiac function. DCM causes congestive heart failure (HF) or critical arrhythmias. Although the overall prognosis has improved, DCM is still considered to have an unsatisfactory prognosis $(1,2)$. An

${ }^{1}$ Division of Cardiovascular Medicine, Department of Internal Medicine, Kobe University Graduate School of Medicine, Japan, ${ }^{2}$ Department of Radiology, Kobe University Graduate School of Medicine, Japan and ${ }^{3}$ Kobe University Graduate School of Health Science, Japan Received for publication November 10, 2014; Accepted for publication December 23, 2014 Correspondence to Dr. Atsushi K. Kono, ringonotegami@mac.com 
older age, greater functional impairment, poorer systolic function of the left ventricle (LV), lower serum sodium concentration, poorer renal function, broader QRS complex, lower blood pressure, and the inability to tolerate diseasemodifying drugs are associated with a poorer prognosis and are mentioned in the guidelines for HF management (3). Lapu-Bula et al. reported 69 cardiac deaths among 197 DCM patients over an average period of 62 years \pm 13 months (4). Among these 69 cardiac deaths, 42 (61\%) were due to progressive pump failure. The stratification of highrisk patients progressing to $\mathrm{HF}$ is important. Compared with the myocardia of healthy controls, the myocardia of patients with chronic LV dysfunction are characterized by significant reductions of pre-synaptic norepinephrine uptake and postsynaptic beta-adrenoceptor densities $(5,6)$. Cardiac sympathetic nerve imaging with I-123 metaiodobenzylguanidine (MIBG) is a noninvasive tool for risk stratification in patients with HF (7). In patients with ischemic or nonischemic cardiomyopathy, cardiac MIBG activity is a very powerful predictor of survival (8). Other studies have previously reported that MIBG schintigraphy could predict the functional improvement in DCM patients $(9,10)$.

Recently, cardiovascular magnetic resonance (CMR) imaging has been recognized as a useful tool to evaluate cardiac diseases. A comprehensive analysis using cine, T1weighted, T2-weighted, perfusion, and late enhancement imaging enables us to assess the function of the heart and the character of the myocardial tissue. T2-weighted imaging (T2WI) is used in the diagnosis of myocardial edema in myocardial infarction (11). However, conventional T2WI with black-blood pulse is susceptible to artifacts, and the analysis of T2WI is subject to the observer's perceptions (12); therefore, quantitative T2 measurement is needed. T2 mapping has been introduced because this method is more quantitative and objective (13). T2 mapping is generated by calculating the decay of the $\mathrm{T} 2$ value on each pixel from more than 2 different images with different echo-times (TEs). T2WI imaging or T2 mapping in cardiomyopathies is of interest; however, there are only a few reports on the utilization of these methods in patients with DCM $(14,15)$. However, the clinical relevance of T2 mapping in DCM patients remains unclear.

We evaluated the efficacy of T2 mapping in DCM patients for the assessment of cardiac dysfunction severity and functional recovery using comparison with MIBG scintigraphy as a reference standard.

\section{Materials and Methods}

Our institutional review board approved this retrospective study and waived written informed consent for the use of the patients' clinical and imaging data.

\section{Study population}

We retrospectively reviewed 22 consecutive idiopathic DCM patients (aged 56.8 \pm 13.4 years; 6 women and 16 men) who underwent CMR, MIBG, and trans-thoracic echocardiography (TTE). The diagnosis of DCM was made according to the World Health Organization/International Society and Federation of Cardiology criteria (16). We excluded patients with ischemic cardiomyopathy, arrhythmias, sarcoidosis, congenital heart diseases, past histories of myocarditis, severe anemia requiring frequent blood transfusions, muscle disorders (such as myositis or muscular dystrophy), and general contraindications for CMR. We also excluded patients with neuro-degenerative diseases, such as Parkinson disease and dementia with Lewy bodies, because of the decreased MIBG uptake in these patients. Additionally, we excluded patients who were on reserpine or tricyclic antidepressants, which are known to affect MIBG uptake. All enrolled patients were admitted to our hospital for detailed examinations and the treatment of HF. Each patient was treated by a clinical cardiologist according to the guidelines for the management of chronic HF in our country (published by the Japanese Circulation Society and available at http:// www.j-circ.or.jp/guideline/pdf/JCS2010_matsuzaki_h.pdf).

\section{CMR acquisition and analysis}

CMR was performed with a 1.5T scanner (Achieva 1.5T, Philips Medical Systems, Best, the Netherlands) and a cardiac 5-channel coil. Cine, T2 mapping, and late gadolinium enhancement (LGE) was routinely obtained. A total of 12 short-axis cine images, which covered both ventricles, were obtained with $8 \mathrm{~mm}$ thicknesses and $2 \mathrm{~mm}$ gaps. We also took 2- and 4-chamber long-axis cine images. T2 mapping was obtained at the apical, mid-ventricular, and basal levels by means of a multi-TE fast spin echo sequence (15). Two different TEs were used: 16-16.5 ms and $100 \mathrm{~ms}$ with electrocardiogram (ECG) gating. Based on the 17-segment model proposed by the American Heart Association (17), we used and evaluated 16 segments (segments 1-6 at the basal, 7-12 at the mid-ventricular, and 13-16 at the apical levels, but not the 17th segment at the apex) for T2 mapping. As we set the region of interest (ROI) on each segment of the myocardium by manually tracing, we measured the mean T2 value for each patient on a general viewer (ShadeQuest, Yokogawa Medical Solutions Corporation, Tokyo, Japan). LGE was performed 10 minutes after the administration of $0.1 \mathrm{mmol} / \mathrm{kg}$ of gadolinium-diethylene triamine pentaacetic acid (DTPA) (Magnevist, Bayer Schering Pharma AG, Berlin, Germany). LGE was obtained with a 3D segmented inversion recovery gradient echo sequence with a $10 \mathrm{~mm}$ thickness and $5 \mathrm{~mm}$ overlap. The inversion time (TI) was adjusted to the normal myocardium using TI scout imaging. LGE was defined as the area showing higher signal intensity [SI $>$ mean +5 standard deviations (SD)] of the null myocardium. Through the use of a commercially available workstation (Ziostation ver. 2.1.7.1., Ziosoft Inc., Tokyo, Japan), the LGE area for each patient was semi-automatically calculated by integrating the LGE area in each slice. Measuring T2 values and LGE area was performed with the consensus readings of a cardiologist (TI, with 6 years of experience) 


\section{Early Images}

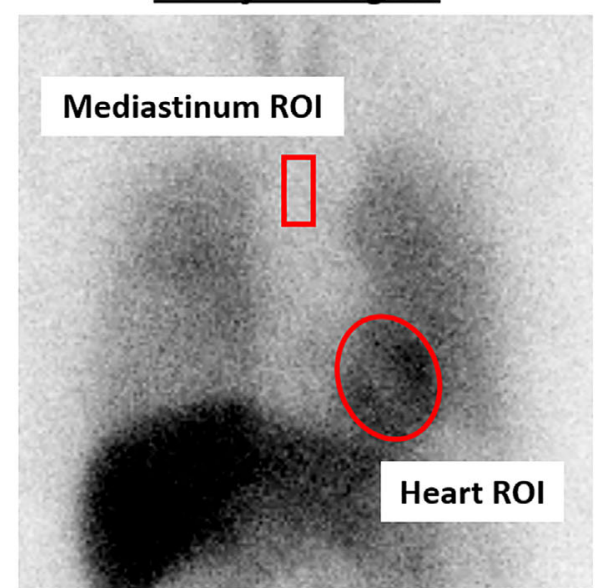

Delayed Images

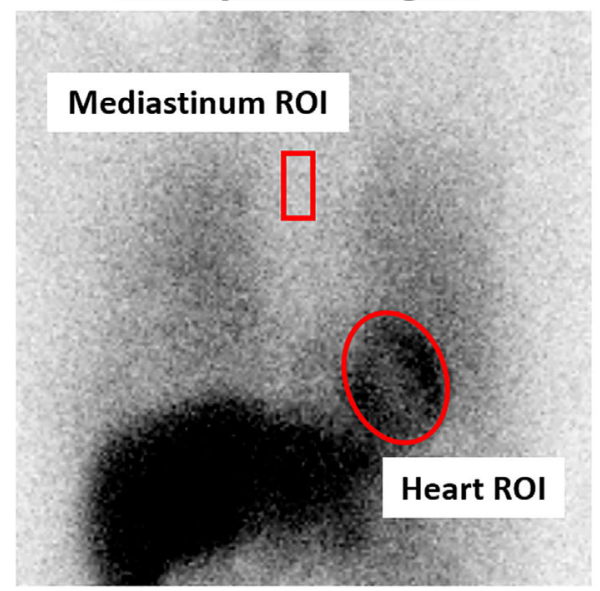

Heart ROI Mean Count: H

Mediastinum Mean Count: M

$$
\text { Washout Rate }=\frac{(\text { Early H }- \text { Early M) }-(\text { Delayed H }- \text { Delayed M) }}{\text { Early H - Early M }} \times 100(\%)
$$

Figure 1. Scheme of the analysis of MIBG scintigraphy. A rectangular ROI was manually placed in the upper mediastinum, and an oval ROI was manually placed on the heart. The heart-to-mediastinum ratio on the early and delayed images was computed, and the washout rate was calculated from the equation presented in the figure.

and a radiologist (AKK, with 11 years of experience).

\section{MIBG acquisition and analysis}

After thyroid blockade by the oral administration of 100 mg of potassium iodide, $111 \mathrm{MBq}$ of MIBG was intravenously injected. At 15 minutes (early phase) and 4 hours (delayed phase) after MIBG injection, a planar anterior image of the chest was acquired with a dual-head rotating gamma camera (Vertex Plus, ADAC Laboratories, Milpitas, USA) equipped with a low-energy, general purpose collimator, and then stored in a $128 \times 128$ matrix. An ROI over the heart $(\mathrm{H})$ was manually drawn and a rectangular ROI over the upper mediastinum (M) was used as the reference background. Mean counts were obtained for each ROI. The heart-to-mediastinum $(\mathrm{H} / \mathrm{M})$ ratios in the early and delayed phases were determined from the anterior planar images (Fig. 1). The washout rate (WOR) was also calculated by the following formula: WOR $=[($ early $\mathrm{H}-$ early $\mathrm{M})-($ delayed $\mathrm{H}$ - delayed M)]/(early H - early M) $\times 100(\%)$.

\section{TTE acquisition and analysis}

The standard TTE was performed using a commercially available echocardiographic system (Vivid 7, GE Vingmed Ultrasound AS, Horten, Norway). Digital routine twodimensional cine loops from 3 consecutive heartbeats were obtained during the end-expiratory breath hold from standard apical views (4-chamber, 2-chamber, and long-axis) and standard LV short-axis views (basal, mid, and apical) at depths of $11-20 \mathrm{~cm}$ (mean, $16 \pm 3 \mathrm{~cm}$ ). The frame rates were

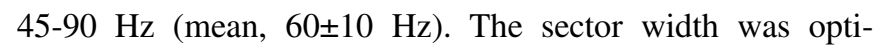

mized to allow complete myocardial visualization while maximizing the frame rate. The LV volumes and ejection fractions (EF) were assessed by means of the biplane Simpson's rule (18). TTE was performed at baseline and the 6month follow-up. The difference of LVEF ( $\triangle \mathrm{LVEF}$ ) was calculated by the following formula: $\triangle \mathrm{LVEF}=(\mathrm{LVEF}$ at follow-up) - (LVEF at baseline). We then classified the patients with $\triangle \mathrm{LVEF} \geq 15 \%$ as responders and the patients with $\triangle \mathrm{LVEF}<15 \%$ as non-responders.

\section{Statistical analysis}

Data are expressed as mean $\pm \mathrm{SD}$, or median [interquartile range (IQR)] for the continuous variables and as absolute numbers for the categorical variables. We used the Mann-Whithey $U$ test or the Welch test to compare the continuous variables and Fisher's exact test to compare the categorical variables. We used the Spearman rank test to analyze correlations. Statistical analyses of the data were performed using JMP software (version 9.0, SAS Institute, Cary, USA). A p value $<0.05$ was considered to be statistically significant.

We conducted the following analyses: 1) the correlation between the T2 values with the MIBG indices and 2) the differences in the T2 values, LGE area, and MIBG indices between the responders and non-responders. 
Table 1. Patient Characteristics.

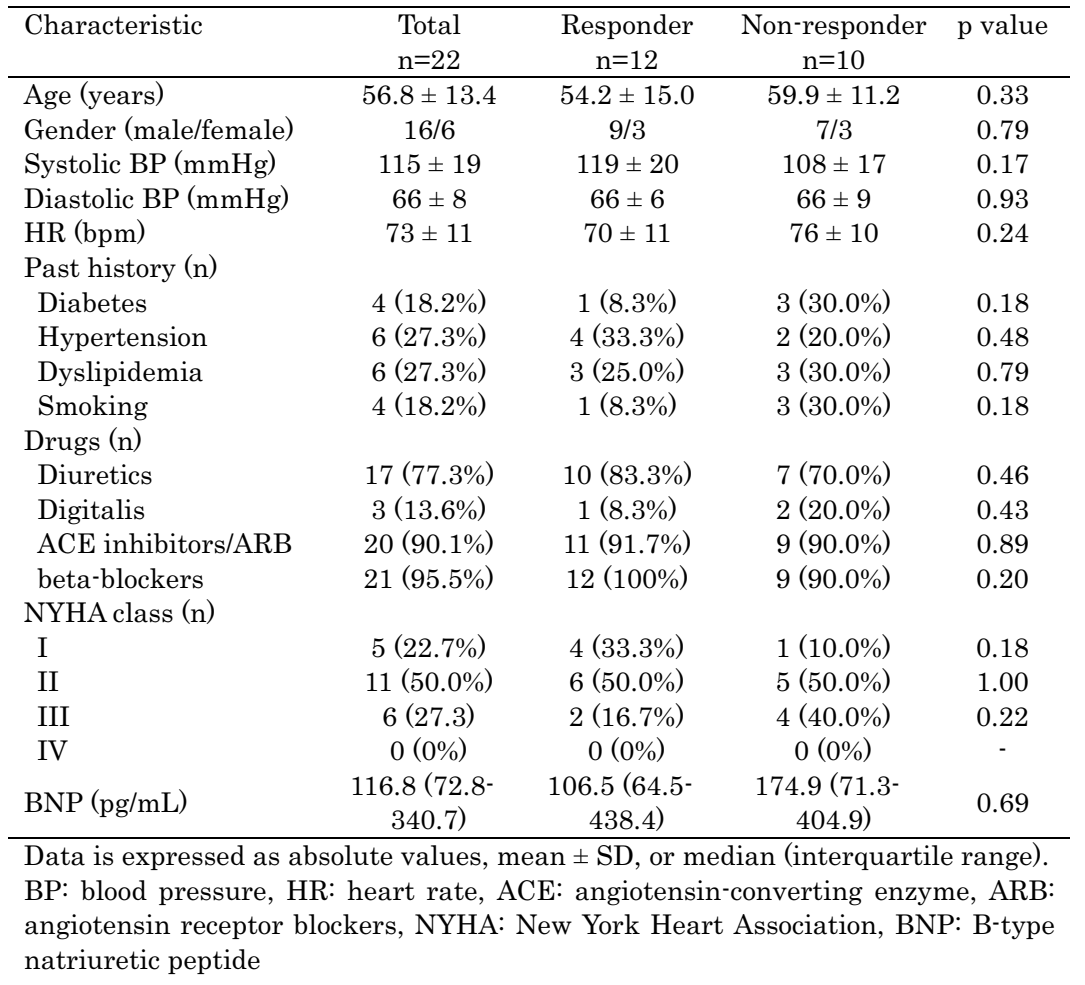

\section{Results}

\section{Patient characteristics, clinical events, and TTE ex- aminations}

The patient characteristics are summarized in Table 1. In all patients, cardiac functional parameters at baseline were as follows: LV end-diastolic volume index (LVEDVI), 109.7 $\pm 34.1 \mathrm{~mL}$; LV end-systolic volume index (LVESVI), $78.7 \pm 30.6 \mathrm{~mL}$; and LVEF, $31.9 \pm 10.7 \%$. The parameters after 6 months were: LVEDVI, $80.7 \pm 40.7 \mathrm{~mL}$; LVESVI, $48.7 \pm 37.9 \mathrm{~mL}$; and LVEF, $46.1 \pm 14.4 \%$. The mean value of $\triangle \mathrm{LVEF}$ was $14.1 \pm 9.6 \%$.

According to the value of $\triangle \mathrm{LVEF}$, the numbers of responders and non-responders were 12 and 10, respectively. There was no difference in the patient characteristics at baseline between the responders and non-responders $(\mathrm{p} \geq 0.05$ for each characteristic). Furthermore, there was no difference in the treatment at baseline and at follow-up between the 2 groups. None of the patients experienced cardiac death, hospitalization, or invasive treatment, such as implantable cardioverter-defibrillator (ICD) or cardiac resynchronization therapy. The functional parameters between the responders and non-responders are also summarized in Table 2 .

\section{T2 mapping, LGE, and MIBG scintigraphy}

Representative images of T2 mapping and MIBG scintigraphy are shown in Fig. 2. The interval between CMR and MIBG scintigraphy was 7 days (IQR 2-10 days).
In all patients, the mean and $\mathrm{SD}$ of the $\mathrm{T} 2$ values were 64.5 $\pm 6.6 \mathrm{~ms}$ (range: 56.2-86.0). The mean and SD of the LGE area was $3.9 \pm 5.0 \%$ (range: 0-19.2). The mean values and SDs of early H/M, delayed H/M, and WOR were 2.06 \pm 0.25 (range: 1.54-2.53), 1.94 \pm 0.35 (range: 1.24-2.54), and $43.5 \pm 11.8 \%$ (range: $27.3-72.4$ ), respectively. The T2 values correlated with early $\mathrm{H} / \mathrm{M}(\rho=-0.43, \mathrm{p}=0.047)$, delayed $\mathrm{H} / \mathrm{M}$ $(\rho=-0.50, p=0.017)$, and WOR $(\rho=0.46, p=0.031)$, respectively (Fig. 3).

The mean T2 values, LGE area, and MIBG indices between the responders and non-responders are summarized in Table 2. The $\mathrm{T} 2$ values were lower in the responders than non-responders $(61.4$ vs. $68.1 \mathrm{~ms}, \mathrm{p}=0.013)$. In contrast, the LGE area did not significantly differ between the responders and non-responders (3.4\% vs. $4.4 \%, \mathrm{p}=0.67)$. Each index of MIBG was better in the responders than non-responders; however, these differences were not significant $(\mathrm{p}=0.16$ for early H/M, 0.07 for delayed H/M, and 0.08 for WOR, respectively).

\section{Discussion}

DCM is a cardiomyopathy causing HF, and MIBG scintigraphy is useful in assessing the severity or prognosis of HF. This study showed that the T2 values correlated with the MIBG indices, and the functional recovery at the 6month follow-up significantly differed between the responders and non-responders.

The normal myocardial $\mathrm{T} 2$ value recently reported was approximately $55 \mathrm{~ms}$ (range 50-58) (12) or 51.2 \pm 1.6 $\mathrm{ms}(15)$ in the $1.5 \mathrm{~T}$ scanner. The myocardial $\mathrm{T} 2$ value in 
Table 2. Results of Echocardiography, MIBG Scintigraphy, and T2 Mapping.

\begin{tabular}{|c|c|c|c|c|}
\hline & Modality & $\begin{array}{c}\text { Responder } \\
\mathrm{n}=12\end{array}$ & $\begin{array}{c}\text { Non-responder } \\
n=10\end{array}$ & $\mathrm{p}$ value \\
\hline \multirow[t]{11}{*}{ Baseline } & Echocardiography & & & \\
\hline & LVEDVI (mL) & $103.9 \pm 32.8$ & $116.6 \pm 36.1$ & 0.40 \\
\hline & LVESVI (mL) & $77.5 \pm 27.5$ & $80.2 \pm 35.5$ & 0.84 \\
\hline & LVEF (\%) & $30.4 \pm 10.4$ & $33.7 \pm 11.3$ & 0.49 \\
\hline & MIBG scintigraphy & & & \\
\hline & H/M (early) & $2.13 \pm 0.18$ & $1.98 \pm 0.29$ & 0.16 \\
\hline & H/M (delayed) & $2.06 \pm 0.27$ & $1.79 \pm 0.40$ & 0.07 \\
\hline & WOR (\%) & $39.5 \pm 7.0$ & $48.2 \pm 14.8$ & 0.08 \\
\hline & $C M R$ & & & \\
\hline & $\mathrm{T} 2$ value $(\mathrm{ms})$ & $61.4 \pm 3.1$ & $68.1 \pm 7.9$ & 0.013 \\
\hline & LGE area (\%) & $3.4 \pm 4.2$ & $4.4 \pm 5.7$ & 0.67 \\
\hline \multirow[t]{5}{*}{ Follow-up } & Echocardiography & & & \\
\hline & LVEDVI (mL) & $71.9 \pm 37.8$ & $91.4 \pm 43.3$ & 0.27 \\
\hline & LVESVI (mL) & $38.2 \pm 31.1$ & $61.3 \pm 43.0$ & 0.16 \\
\hline & LVEF (\%) & $52.2 \pm 11.5$ & $38.9 \pm 14.8$ & 0.027 \\
\hline & $\triangle \mathrm{LVEF}(\%)$ & $21.7 \pm 3.9$ & $5.2 \pm 5.5$ & - \\
\hline
\end{tabular}

Data is expressed mean \pm SD.

LV: left ventricle, EDVI: end-diastolic volume index, ESVI: end-systolic volume index, EF: ejection fraction, MIBG: metaiodobenzylguanidine, H/M: heart-to-mediastinum count ratio, WOR: washout rate, CMR: cardiovascular magnetic resonance imaging, LGE: late gadolinium enhancement, $\triangle \mathrm{LVEF}$ : the difference of LVEF calculated as $\triangle \mathrm{LVEF}=\mathrm{LVEF}$ at follow-up-LVEF at baseline
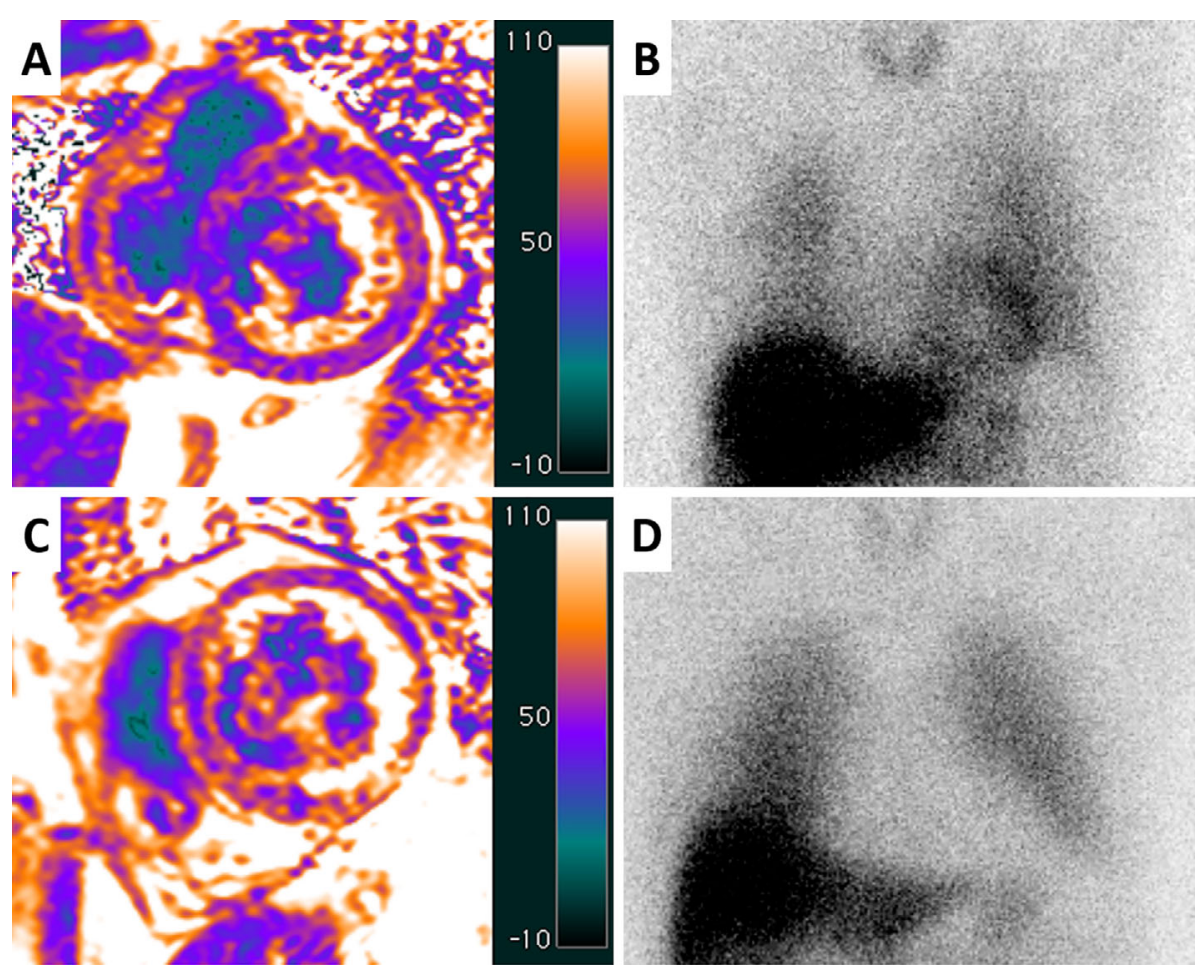

Figure 2. Representative images of T2 mapping and MIBG scintigraphy of DCM patients. Images of T2 mapping and MIBG scintigraphy in the delayed phase of a responder (53-year-old man) (A and B) and a non-responder (64-year-old man) (C and D) are shown. In the responder, T2 mapping showed a normal T2 value of $58.1 \mathrm{~ms}$ (purple color in A), and MIBG scintigraphy showed a normal heart-to-mediastinum ratio (H/M) of 2.5 (B). In the non-responder, T2 mapping showed a diffusely increased T2 value of $70.1 \mathrm{~ms}$ (orange color in C), and MIBG scintigraphy showed decreased myocardial uptake and a decreased H/M of 1.3 (D).

DCM was previously reported as $61 \pm 0.4 \mathrm{~ms}$ for the mild cardiac dysfunction group (LVEF $>35 \%$ ) and $67 \pm 6.8 \mathrm{~ms}$ for the severe dysfunction group (LVEF $\leq 35 \%)$ (15). Our result $(64.5 \pm 6.6 \mathrm{~ms})$ was comparable to the previous report. 

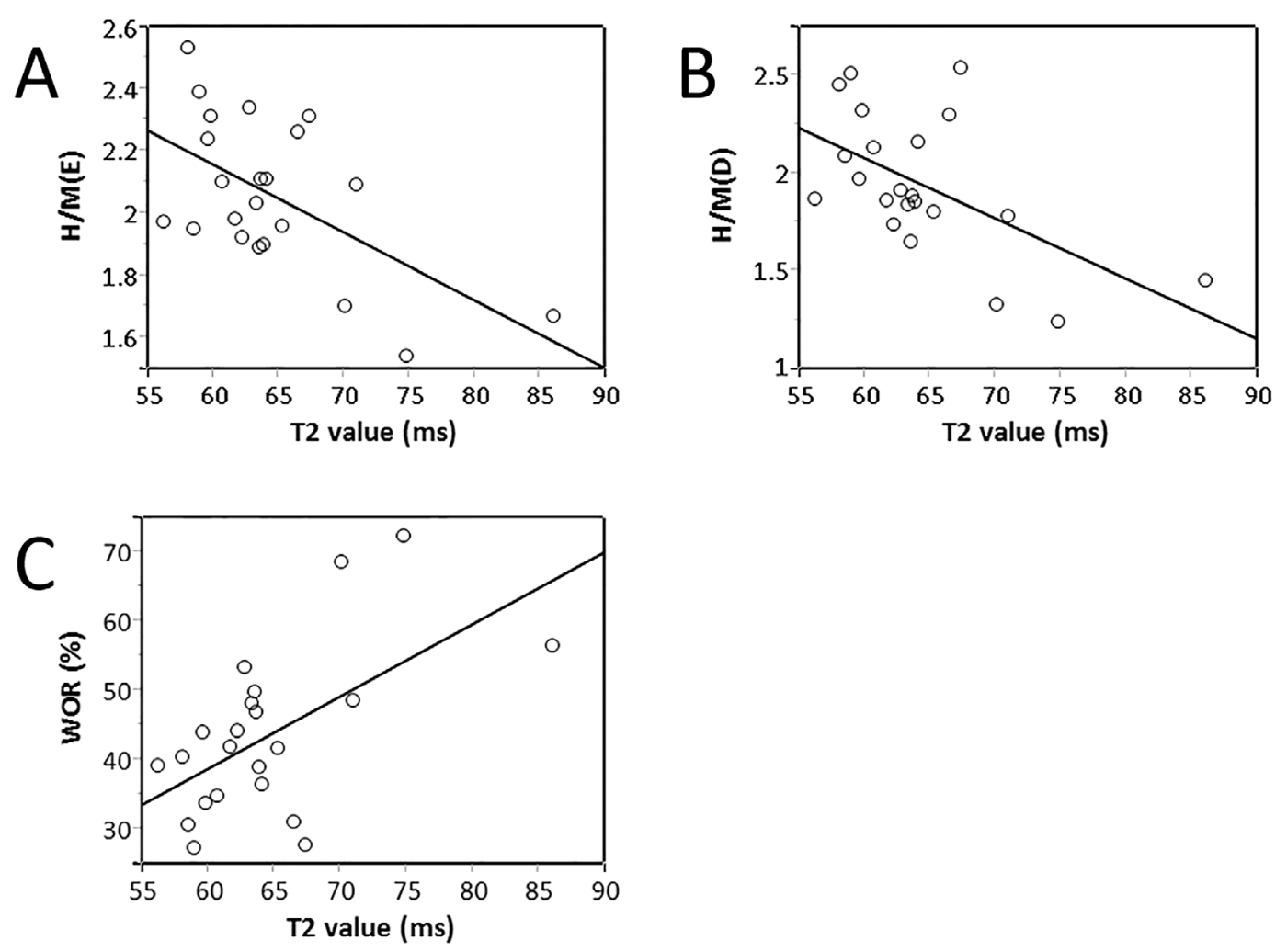

Figure 3. Scatterplots of the T2 value with MIBG indices. The T2 value was plotted with the heartto-mediastinum ratio (H/M) in the early phase [H/M (E), A], H/M in the delayed phase [H/M (D), B], and the washout rate (WOR, C). Correlation analyses indicated that the T2 value correlated with H/M (E) $(\rho=-\mathbf{0 . 4 3}, \mathbf{p = 0 . 0 4 7}), \mathrm{H} / \mathrm{M}(\mathrm{D})(\rho=-\mathbf{0 . 5 0}, \mathbf{p}=\mathbf{0 . 0 1 7})$, and WOR $(\rho=0.46, p=0.031)$.

MIBG scintigraphy is a semi-quantitative analysis; therefore, as the uptake decreases commensurate with the disease severity, setting ROI on the heart becomes more difficult due to the increased uptake in the lungs. Therefore, the MIBG indices can underestimate the severity in such cases. Because T2 mapping is quantitative, this method is considered to be superior. Conversely, T2 mapping is less useful for arrhythmias because TR is not constant due to the irregular ECG gating. Thus, for HF patients with arrhythmias, MIBG scintigraphy is considered to be superior.

MIBG scintigraphy with the indices of $\mathrm{H} / \mathrm{M}$ and WOR is now widely used to evaluate the sympathetic nerve activity. The early $\mathrm{H} / \mathrm{M}$ reflects the distribution of cardiac sympathetic nerve and uptake- 1 function in the sympathetic nerve terminals. The delayed $\mathrm{H} / \mathrm{M}$ provides additional information of the MIBG washout in the early H/M. WOR reflects the degree of sympathetic drive. A decreased $\mathrm{H} / \mathrm{M}$ and increased WOR are associated with increased sympathetic activity (19). The value of $\mathrm{H} / \mathrm{M}$ was found to correlate significantly with the myocardial content of noradrenaline, suggesting that a decrease in the MIBG uptake is related to sympathetic nerve dysfunction (20). Sympathetic nerve dysfunction was also found to correlate with the myocardial contractile reserve in DCM patients (21); thus, MIBG scintigraphy can assess the severity of myocardial dysfunction. MIBG scintigraphy is also used to predict the prognosis. When the value of $\mathrm{H} / \mathrm{M}$ decreased or WOR increased, the patients showed significantly poorer prognoses $(8,22,23)$. Additionally, MIBG scintigraphy was useful in predicting the functional improvement for the treatment $(9,10)$. In this study, the T2 values derived from T2 mapping correlated with the MIBG indices; therefore, T2 mapping may be able to assess the HF severity. Moreover, because the T2 values significant differed between the responders and nonresponders, T2 mapping may be useful for predicting the functional recovery in DCM.

The physiology between decreasing MIBG uptake and increasing $\mathrm{T} 2$ values should be different. The rationale for why the $\mathrm{T} 2$ values correlated with the MIBG severity is not clear; however, myocardial damage may explain this correlation. As the myocardium is damaged, the $\mathrm{T} 2$ value increases and MIBG uptake decreases. Additionally, Turpeinen et al. found that the cardiac sympathetic activity correlated with inflammatory markers by comparing MIBG indices and interleukin-6 (IL-6) (a proinflammatory cytokine) (24). They concluded that myocardial sympathetic innervation and activity are related to inflammation in DCM. This relationship may also explain the observations of decreased MIBG uptake and increased $\mathrm{T} 2$ values.

The presence of edema or inflammation is generally considered as the central factor influencing $\mathrm{T} 2$ weighted imaging $(11,13)$. If $\mathrm{T} 2$ mapping only reflects the degree of edema, it would not be able to predict the functional recovery. Thus, we presumed that the inflammation may be one 
factor affecting the T2 values in DCM. Chronic or persistent inflammation in the myocardium plays a role in DCM as a cause of disease pathogenesis or progression (25). The abovementioned paper by Turpeinen et al. (24) may partially support the correlation between T2 value and MIBG indices and predict the functional recovery observed in our study.

Previous papers have reported the importance of myocardial fibrosis, as detected by LGE, in DCM patients (26-28). The presence of LGE showed higher mortality, hospitalization, and the requirement of invasive treatments, such as ICD. However, we did not find any significant difference of LGE area in the LV myocardium between the responders and non-responders. The short follow-up period or the small number of the patients in our study may explain the discrepancy between this study and previous reports to some extent. Additionally, current LGE imaging utilizes the nullpoint method. The normal myocardium is set as null when using inversion-recovery pulse; however, this has an intrinsic limitation and should not be used in diffusely diseased cardiomyopathies, such as DCM. This is because the normal null myocardium would not be present. This limitation may result in the presence of only $35-42 \%$ of LGE, even in a population with severe dysfunction $(26,27)$. Although the recent development of quantitative T1 mapping or extracellular volume measurement may help overcome this limitation (29), we did not have these sequences or algorithms. Additionally, the renal function decreases in $\mathrm{HF}$ patients (30), which may make it difficult to administer the gadolinium-based contrast agent. Because of these limitations, T2 mapping has advantages over LGE imaging.

\section{Limitations}

There were several limitations to this study. First, we only included a small number of patients. Because we excluded patients with arrhythmias, a considerable number of DCM patients may have been excluded in this study. We could not perform a prognostic evaluation due to the small sample size. Second, the study was retrospective. Thus, the treatment received may be inhomogeneous in our population. Third, the laboratory data on inflammatory markers, such as high sensitive C-reactive protein or IL-6, were not available because they are not routinely obtained. Further prospective studies including a larger number of patients and more homogenous data and treatments are needed to ascertain the efficacy of T2 mapping.

\section{Conclusion}

T2 mapping in DCM patients was found to correlate with the MIBG indices. Moreover, the T2 values were significantly lower in the responders than non-responders. Therefore, T2 mapping may be useful in assessing the cardiac function and functional recovery in DCM patients.

The authors state that they have no Conflict of Interest (COI).

\section{References}

1. Felker GM, Thompson RE, Hare JM, et al. Underlying causes and long-term survival in patients with initially unexplained cardiomyopathy. N Engl J Med 342: 1077-1084, 2000.

2. Matsumura Y, Takata J, Kitaoka H, et al. Long-term prognosis of dilated cardiomyopathy revisited: an improvement in survival over the past 20 years. Circ J 70: 376-383, 2006.

3. Dickstein K, Cohen-Solal A, Filippatos G, et al. ESC guidelines for the diagnosis and treatment of acute and chronic heart failure 2008: the Task Force for the diagnosis and treatment of acute and chronic heart failure 2008 of the European Society of Cardiology. Developed in collaboration with the Heart Failure Association of the ESC (HFA) and endorsed by the European Society of Intensive Care Medicine (ESICM). Eur J Heart Fail 10: 933-989, 2008.

4. Lapu-Bula R, Robert A, De Kock M, et al. Risk stratification in patients with dilated cardiomyopathy: contribution of Dopplerderived left ventricular filling. Am J Cardiol 82: 779-785, 1998.

5. Ungerer M, Böhm M, Elce JS, Erdmann E, Lohse MJ. Altered expression of beta-adrenergic receptor kinase and beta 1-adrenergic receptors in the failing human heart. Circulation 87: 454-463, 1993.

6. Caldwell JH, Link JM, Levy WC, Poole JE, Stratton JR. Evidence for pre- to postsynaptic mismatch of the cardiac sympathetic nervous system in ischemic congestive heart failure. J Nucl Med 49: 234-241, 2008.

7. Yamashina S, Yamazaki J. Neuronal imaging using SPECT. Eur J Nucl Med Mol Imaging 34: S62-S73, 2007.

8. Merlet P, Valette H, Dubois-Randé JL, et al. Prognostic value of cardiac metaiodobenzylguanidine imaging in patients with heart failure. J Nucl Med 33: 471-477, 1992.

9. Suwa M, Otake Y, Moriguchi A, et al. Iodine-123 metaiodobenzylguanidine myocardial scintigraphy for prediction of response to beta-blocker therapy in patients with dilated cardiomyopathy. Am Heart J 133: 353-358, 1997.

10. Kakuchi H, Sasaki T, Ishida Y, Komamura K, Miyatake K. Clinical usefulness of 123I meta-iodobenzylguanidine imaging in predicting the effectiveness of beta blockers for patients with idiopathic dilated cardiomyopathy before and soon after treatment. Heart 81: 148-152, 1999.

11. Verhaert D, Thavendiranathan $P$, Giri $S$, et al. Direct $T 2$ quantification of myocardial edema in acute ischemic injury. JACC Cardiovasc Imaging 4: 269-278, 2011.

12. Giri S, Chung YC, Merchant A, et al. T2 quantification for improved detection of myocardial edema. J Cardiovasc Magn Reson 11: 56, 2009.

13. Thavendiranathan $P$, Walls $M$, Giri $S$, et al. Improved detection of myocardial involvement in acute inflammatory cardiomyopathies using T2 mapping. Circ Cardiovasc Imaging 5: 102-110, 2012.

14. Jeserich M, Föll D, Olschewski M, et al. Evidence of myocardial edema in patients with nonischemic dilated cardiomyopathy. Clin Cardiol 35: 371-376, 2012.

15. Nishii T, Kono AK, Shigeru M, et al. Cardiovascular magnetic resonance T2 mapping can detect myocardial edema in idiopathic dilated cardiomyopathy. Int J Cardiovasc Imaging 30: 65-72, 2014.

16. Richardson P, McKenna W, Bristow M, et al. Report of the 1995 World Health Organization/International Society and Federation of Cardiology Task Force on the Definition and Classification of cardiomyopathies. Circulation 93: 841-842, 1996.

17. Cerqueira MD, Weissman NJ, Dilsizian V, et al. Standardized myocardial segmentation and nomenclature for tomographic imaging of the heart. A statement for healthcare professionals from the Cardiac Imaging Committee of the Council on Clinical Cardiology of the American Heart Association. Circulation 105: 539-542, 2002. 
18. Lang RM, Bierig M, Devereux RB, et al. Recommendations for chamber quantification: a report from the American Society of Echocardiography's Guidelines and Standards Committee and the Chamber Quantification Writing Group, developed in conjunction with the European Association of Echocardiography, a branch of the European Society of Cardiology. J Am Soc Echocardiogr 18: 1440-1463, 2005.

19. Agostini D, Carrio I, Verberne HJ. How to use myocardial 123IMIBG scintigraphy in chronic heart failure. Eur J Nucl Med Mol Imaging 36: 555-559, 2009.

20. Schofer J, Spielmann R, Schuchert A, Weber K, Schlüter M Iodine-123 meta-iodobenzylguanidine scintigraphy: a noninvasive method to demonstrate myocardial adrenergic nervous system disintegrity in patients with idiopathic dilated cardiomyopathy. J Am Coll Cardiol 12: 1252-1258, 1988.

21. Ohshima S, Isobe S, Izawa $\mathrm{H}$, et al. Cardiac sympathetic dysfunction correlates with abnormal myocardial contractile reserve in dilated cardiomyopathy patients. J Am Coll Cardiol 46: 2061-2068, 2005.

22. Merlet $P$, Benvenuti $C$, Moyse $D$, et al. Prognostic value of MIBG imaging in idiopathic dilated cardiomyopathy. J Nucl Med 40: 917-923, 1999.

23. Imamura $Y$, Fukuyama $T$, Mochizuki T, Miyagawa M, Watanabe $\mathrm{K}$, Investigators EMHFS. Prognostic value of iodine-123metaiodobenzylguanidine imaging and cardiac natriuretic peptide levels in patients with left ventricular dysfunction resulting from cardiomyopathy. Jpn Circ J 65: 155-160, 2001.

24. Turpeinen AK, Vanninen E, Magga $J$, et al. Cardiac sympathetic activity is associated with inflammation and neurohumoral activation in patients with idiopathic dilated cardiomyopathy. Clin Physiol Funct Imaging 29: 414-419, 2009.

25. De Gennaro L, Brunetti ND, Cuculo A, Pellegrino PL, Di Biase M. Systemic inflammation in nonischemic dilated cardiomyopathy. Heart Vessels 23: 445-450, 2008.

26. Assomull RG, Prasad SK, Lyne J, et al. Cardiovascular magnetic resonance, fibrosis, and prognosis in dilated cardiomyopathy. $\mathrm{J}$ Am Coll Cardiol 48: 1977-1985, 2006.

27. Wu KC, Weiss RG, Thiemann DR, et al. Late gadolinium enhancement by cardiovascular magnetic resonance heralds an adverse prognosis in nonischemic cardiomyopathy. J Am Coll Cardiol 51: 2414-2421, 2008.

28. Lehrke S, Lossnitzer D, Schöb M, et al. Use of cardiovascular magnetic resonance for risk stratification in chronic heart failure: prognostic value of late gadolinium enhancement in patients with non-ischaemic dilated cardiomyopathy. Heart 97: 727-732, 2011.

29. Maestrini V, Treibel TA, White SK, Fontana M, Moon JC. T1 mapping for characterization of intracellular and extracellular myocardial diseases in heart failure. Curr Cardiovasc Imaging Rep 7: 9287, 2014.

30. Smith GL, Vaccarino V, Kosiborod M, et al. Worsening renal function: what is a clinically meaningful change in creatinine during hospitalization with heart failure? J Card Fail 9: 13-25, 2003.

(C) 2015 The Japanese Society of Internal Medicine http://www.naika.or.jp/imonline/index.html 